\title{
Le coût est un invariant isopérimétrique
}

\author{
Mikaël Pichot et Stéphane Vassout
}

Résumé. Soit $R$ une relation d'équivalence mesurée ergodique de type $\mathrm{II}_{1}$ sur un espace de probabilité $(X, \mu)$ sans atome. On montre que

$$
C(R)=1+\frac{1}{2} h(R),
$$

où $C(R)$ est le coût de $R$, supposé fini, et $h(R)$ sa constante isopérimétrique. Cela fait suite aux résultats récents de Russell Lyons et des auteurs sur le sujet.

Abstract. Let $R$ be an ergodic measured equivalence relation of type $\mathrm{II}_{1}$ on a probability space $(X, \mu)$ without atom. We show that

$$
C(R)=1+\frac{1}{2} h(R)
$$

where $C(R)$ is the cost of $R$, assumed to be finite, and $h(R)$ its isoperimetric constant. This is a continuation of recent work of Russell Lyons and the authors on the subject.

Mathematics Subject Classification (2010). 20F65.

Keywords. Measured equivalence relations, cost, isoperimetry.

\section{Introduction}

Soit $R$ une relation d'équivalence mesurée ergodique de type $\mathrm{II}_{1}$ (à classes dénombrables) sur un espace de probabilité $(X, \mu)$ sans atome. Comme pour les groupes dénombrables, on associe à tout système générateur $\Phi$ de $R$ un graphe de Cayley $\Sigma_{\Phi}$ sur lequel la relation $R$ agit ( $\Phi$ est aussi appelé un graphage de $R$ ). Suivant G. Levitt (voir [1]), le coût de $R$ est défini par

$$
C(R)=\inf _{\Phi} \frac{1}{2} \operatorname{val}_{\mu}\left(\Sigma_{\Phi}\right)
$$

où l'infimum porte sur les systèmes générateurs de $R$ et val $\mathrm{l}_{\mu}$ est la valence moyenne dans $\Sigma_{\Phi}$ prise relativement à $\mu$. Il s'agit a priori d'un nombre réel compris entre 1 
et $\infty$ (inclus) ; D. Gaboriau a montré dans [1] que toutes les valeurs de $[1, \infty]$ étaient atteintes.

Un autre invariant de $R$, la constante isoperimétrique $h(R)$, peut être défini à partir $\operatorname{des} \Sigma_{\Phi}$ :

$$
h(R)=\inf _{\Phi} h\left(\Sigma_{\Phi}\right)
$$

où $h\left(\Sigma_{\Phi}\right)$ est la constante isopérimétrique de $\Sigma_{\Phi}$, définie de façon appropriée (une définition précise est rappelée à la section 2). Cet invariant, à valeurs dans $[0, \infty]$, a été introduit par R. Lyons et les auteurs dans [3].

Dans [3] il est montré que

$$
C(R) \leqslant 1+\frac{1}{2} h(R),
$$

si $R$ est de coût fini, et que $h(R)=0$ dès que $C(R)=1$. Nous renvoyons à cet article pour des détails sur ces deux énoncés ainsi que leurs preuves. Le théorème principal de cet article complète ces deux résultats :

Théorème 1. Soit $R$ une relation d'équivalence mesurée ergodique de type $\mathrm{II}_{1}$ sur un espace de probabilité $(X, \mu)$ sans atome. Si $C(R)<\infty$, alors

$$
C(R)=1+\frac{1}{2} h(R),
$$

où $C(R)$ est le coût de $R$ et $h(R)$ la constante isopérimétrique.

Le théorème 1 établit l'égalité entre deux invariants de $R$ de nature très différente : le coût de $R$ est un invariant local des graphes de Cayley de $R$ (par définition), alors que la constante isopérimétrique en est un invariant asymptotique. L'énoncé correspondant en théorie des groupes n'est bien sûr pas correct. Si $\Gamma$ est un groupe dénombrable infini de type fini, l'analogue du coût est le nombre minimal $g(\Gamma)$ de générateurs de $\Gamma$ (i.e. la valence minimale des graphes de Cayley divisée par 2), et la constante isopérimétrique de $\Gamma$ est l'infimum $h(\Gamma)$, sur les graphes de Cayley $Y$ de type fini de $\Gamma$, de la constante de isopérimétrique habituelle de $Y$. En général, les deux constantes $g(\Gamma)$ et $h(\Gamma)$ ne coïncident pas modulo renormalisations. Par exemple pour les groupes abéliens libres $\mathbb{Z}^{n}, n \geqslant 2$, ou plus généralement pour les groupes moyennables non cycliques, on a $h(\Gamma)=0$ et $g(\Gamma)>1$. Une égalité analogue à celle du théorème 1 a lieu cependant pour le groupe libre $\Gamma=F_{k}$ à $k$ générateurs; dans ce cas $g\left(F_{k}\right)=k$ et $h\left(F_{k}\right)=2 k-2$. En fait, il est facile de voir que pour un groupe de type fini $\Gamma$, l'égalité $g(\Gamma)=1+\frac{1}{2} h(\Gamma)$ n'a lieu que si $\Gamma$ est un groupe libre. En effet, $g(\Gamma)$ est atteint pour un certain système générateur (fini) $S$, et pour ce $S$ on a donc $2(|S|-1)=h(\Gamma) \leqslant h(Y)$, où $h(Y)$ est la constante isopérimétrique du graphe de Cayley $Y$ de $\Gamma$ relativement à $S$. En appliquant cette inégalité aux boules $B_{n}$ de $Y$, on obtient par un calcul immédiat que $\left|B_{n}\right| \geqslant 2|S|(2|S|-1)^{n-1}$ pour tout $n \geq 1$, et 
donc que $\Gamma$ est un groupe libre. Au contraire, le coût $C(R)$ de $R$ n'est jamais atteint, sauf si $R$ est arborable (cf. [1]).

Remerciements. Nous remercions George Skandalis qui a suggéré que l'inégalité de [3] était une égalité. Le premier auteur est financé par JSPS et l'Université de Tokyo (IPMU).

\section{Rappels et notations}

Faisons quelques brefs rappels pour fixer les notations. Pour plus de détails sur les graphages et le coût des relations d'équivalences, nous renvoyons aux articles [1], [2] de D. Gaboriau.

Soit $\Phi$ une famille dénombrable d'isomorphismes partiels de $R$, i.e. d'isomorphismes partiels $\varphi$ de $X$ tels que $x \sim \varphi(x) \bmod (R)$. Le coût de $\Phi$ est le nombre positif (éventuellement infini)

$$
C(\Phi)=\sum_{\varphi \in \Phi} \mu(\operatorname{dom} \varphi)=\sum_{\varphi \in \Phi} \mu(\operatorname{Im} \varphi)
$$

où dom $\varphi$ et $\operatorname{Im} \varphi$ sont respectivement le domaine et l'image de $\varphi$. On dit que $\Phi$ est un système générateurs de $R$ si, presque sûrement, étant donnés deux points $x, y \in X$ équivalents modulo $R$, il existe une composée $\psi=\varphi_{n}^{\varepsilon_{n}} \ldots \varphi_{1}^{\varepsilon_{1}}$ telle que $\psi(x)=y$, où $\varphi_{i} \in \Phi$ et $\varepsilon_{i} \in\{-1,1\}$. Le coût de $R$ est l'infimum

$$
C(R)=\inf _{\Phi} C(\Phi)
$$

pris sur tous les systèmes générateurs $\Phi$ de $R$ (voir [1]). À un système générateur $\Phi$ de $R$ est naturellement associé un graphe de Cayley $\Sigma_{\Phi}$ de $R$. Rappelons simplement que $\Sigma_{\Phi}$ est fibré au-dessus de $X$, et que la fibre $\Sigma_{\Phi}^{x}$ en $x \in X$ est un graphe connexe dont les sommets sont les éléments de $R^{x}$ et les arêtes l'ensemble des couples $\left((x, y),(x, \varphi(y)) \in R^{x} \times R^{x}\right.$ pour $\varphi \in \Phi$ tel que $y \in \operatorname{dom} \varphi$. Il est clair que la définition du coût de $R$ donné ci-dessus coïncide avec celle donnée en introduction.

Dans [3] nous définissons une constante isopérimétrique $h\left(\Sigma_{\Phi}\right)$ de $\Sigma_{\Phi}$ comme suit :

$$
h\left(\Sigma_{\Phi}\right)=\inf _{A} \frac{v^{(1)}\left(\partial_{\Sigma_{\Phi}} A\right)}{|A|}
$$

où $v^{(1)}$ est la mesure de décompte sur le 1-squelette de $\Sigma_{\Phi}$, et $A$ un ensemble fini de sommets symétriques de $\Sigma_{\Phi}$ deux à deux disjoints. Par définition, on appelle sommet symétrique de $\Sigma_{\Phi}$ (le graphe d') un automorphisme du groupe plein de $R$ (i.e. un automorphisme de $X$ dont le graphe est inclus dans $R$ ), et on dit que deux sommets symétriques sont disjoints si l'intersection de leurs graphes est négligeable pour la mesure sur $R$ (voir [3], Definition 4.1). Le bord $\partial_{\Sigma_{\Phi}} A$ de l'ensemble $A$ est 
l'ensemble des arêtes de $\Sigma_{\Phi}$ avec exactement un sommet dans $A$ (i.e. dans le graphe d'un automorphisme de $A$ ). Notons que cette définition de $h\left(\Sigma_{\Phi}\right)$ ne revient pas à «moyenner la constante isopérimétrique des fibres».

La constante isopérimétrique de $R$ est l'infimum

$$
h(R)=\inf _{\Phi} h\left(\Sigma_{\Phi}\right)
$$

pris sur tous les systèmes générateurs $\Phi$ de $R$.

\section{Démonstration du théorème 1}

Soit $R$ une relation d'équivalence ergodique de type $\mathrm{II}_{1}$ sur un espace de probabilité $(X, \mu)$ sans atome. Nous montrons ici que

$$
C(R) \geqslant 1+\frac{1}{2} h(R),
$$

l'inégalité inverse faisant l'objet de [3], Theorem 12. On suppose $C(R)<\infty$.

Soit $\varphi$ un automorphisme ergodique du groupe plein de $R$. Étant donné $\varepsilon>0$, on peut compléter $\varphi$ par une famille $\Phi_{\varepsilon}^{\prime}$ d'isomorphismes partiels de $R$ de sorte que

$$
\Phi_{\varepsilon}=\Phi_{\varepsilon}^{\prime} \cup\{\varphi\}
$$

soit un graphage de $R$, satisfaisant

$$
C\left(\Phi_{\varepsilon}\right)<C(R)+\varepsilon / 4
$$

(voir [1], Lemme III.5).

Soit $f$ la fonction définie pour $x \in X$ par

$$
f(x)=\sum_{\psi \in \Phi_{\varepsilon}^{\prime}} \chi_{\operatorname{dom} \psi}(x)+\chi_{\operatorname{Im} \psi}(x) .
$$

Elle a pour intégrale

$$
\int_{X} f \mathrm{~d} \mu=2 C\left(\Phi_{\varepsilon}^{\prime}\right)
$$

Comme la mesure $\mu$ est invariante, il en résulte que

$$
\frac{1}{n+1} \int_{X} \sum_{i=0}^{n} f\left(\varphi^{i}(x)\right) \mathrm{d} \mu(x)=2 C\left(\Phi_{\varepsilon}^{\prime}\right)
$$

pour tout nombre entier $n \geqslant 0$.

Fixons $n>4 / \varepsilon$ et considérons l'ensemble $A_{n}$ défini par

$$
A_{n}=\left\{\varphi^{i}\right\}_{i=0}^{n} .
$$


Clairement $A_{n}$ est un ensemble de points symétriques deux à deux disjoints de $\Sigma_{\varepsilon}$, au sens de [3, Definition 8]. De plus pour $x \in X$ le bord de $A_{n}^{x}$ dans $\Sigma_{\varepsilon}^{x}$ vérifie

$$
\left|\partial_{\Sigma_{\varepsilon}^{x}} A_{n}^{x}\right| \leqslant \sum_{i=0}^{n} f\left(\varphi^{i}(x)\right)+2
$$

où $|\cdot|$ est le cardinal. Notons qu'on a égalité si $x \not \psi(x) \bmod \left(R_{\varphi}\right)$ pour tout $\psi \in \Phi_{\varepsilon}^{\prime}$ et tout $x \in \operatorname{dom} \psi-$ ce qu'on peut supposer a priori quitte à réduire les domaines des éléments de $\Phi_{\varepsilon}^{\prime}$, mais ce n'est pas nécessaire.

Par suite on a :

$$
\frac{1}{n+1} v^{(1)}\left(\partial_{\Sigma_{\varepsilon}} A_{n}\right) \leqslant 2 C\left(\Phi_{\varepsilon}^{\prime}\right)+\frac{2}{n+1}<2(C(R)-1)+\varepsilon,
$$

où $v^{(1)}$ est la mesure naturelle sur le 1-squelette de $\Sigma_{\varepsilon}$ (associée à la mesure de Haar sur $R$ ). Comme

$$
h(R) \leqslant \inf _{A} \frac{v^{(1)}\left(\partial_{\Sigma_{\varepsilon}} A\right)}{|A|} \leqslant \frac{1}{n+1} v^{(1)}\left(\partial_{\Sigma_{\varepsilon}} A_{n}\right),
$$

on obtient

$$
h(R)<2(C(R)-1)+\varepsilon
$$

et lorsque $\varepsilon \rightarrow 0$,

$$
C(R) \geqslant 1+\frac{1}{2} h(R)
$$

ce qu'il fallait démontrer.

\section{Commentaires}

4.1. Formule de compression. Dans [3] on montre que le groupe fondamental d'une relation uniformément non moyennable ergodique $R$ est trivial, en établissant l'inégalité

$$
h(R) \leqslant \mu(D) h\left(\left.R\right|_{D}\right)
$$

où $D$ est une partie borélienne non négligeable de $X$ et $\left.R\right|_{D}$ est la restriction de $R$ à $D$. En fait cette inégalité est une égalité :

Corollaire 2. Soit $R$ comme dans le théorème 1 et $D$ une partie borélienne non négligeable de X. On a

$$
h(R)=\mu(D) h\left(\left.R\right|_{D}\right)
$$

Ceci résulte immédiatement du théorème 1 et la proposition II.6 de [1] concernant le coût. 
4.2. Une autre définition du bord. Conservant les notations introduites ci-dessus, on peut aussi définir le bord $\partial_{\Sigma} A$ comme l'ensemble des points de $R$ dans le bord de $A$ relativement à $\Sigma$ (plutôt que les arêtes de $\Sigma$ dans le bord de $A$ relativement à $\Sigma$ ). On obtient alors une constante $h^{\prime}(R)$, a priori plus petite que $h(R)$, et les résultats de [3] établissent l'inégalité (voir la note p. 598)

$$
C(R) \leqslant 1+\frac{h^{\prime}(R)}{2} .
$$

On a donc en fait, d'après le théorème 1 :

$$
C(R)=1+\frac{h(R)}{2}=1+\frac{h^{\prime}(R)}{2} .
$$

Comme noté dans [3], p. 598, la version $h^{\prime}$ de la constante isopérimétrique est évidemment majorée par le taux de croissance uniforme $\omega(\Gamma)$ de $\Gamma$ moins 1 , et on a donc

$$
\omega(\Gamma) \geqslant 2 \beta_{1}(\Gamma)+1
$$

(voir [3]), Cor. 3.2, où $\beta_{1}(\Gamma)$ est le premier nombre de Betti $\ell^{2}$ de $\Gamma$.

4.3. Taux de cycles. Rappelons qu'à une relation d'équivalence $R$ ergodique de type $\mathrm{II}_{1}$ est associée une suite de nombres positifs $\beta_{0}(R), \beta_{1}(R), \beta_{2}(R), \ldots$, appelés nombres de Betti $\ell^{2}$ de $R$ (voir [2]). On a toujours

$$
C(R) \geqslant \beta_{1}(R)+1
$$

et on se sait pas si l'inégalité peut être stricte (voir la section 3.6 de [2]). Le taux de cycle de $R$ (voir [4]) est défini comme suit :

$$
\tau(R)=\inf _{\Phi} \operatorname{dim}_{L R} \overline{Z_{1}\left(\Sigma_{\Phi}\right)},
$$

où l'infimum est pris sur les systèmes générateurs $\Phi$ de $R, \Sigma_{\Phi}$ est le graphe de Cayley de $\Phi, Z_{1}\left(\Sigma_{\Phi}\right)$ est l'espace des cycles de $\Sigma_{\Phi}$. La dimension $\operatorname{dim}_{L R}$ est la dimension de l'adhérence Hilbertienne $\overline{Z_{1}\left(\Sigma_{\Phi}\right)}$ prise au sens de Murray et von Neumann (comme pour les nombres de Betti $\ell^{2}$ ), où $L R$ désigne l'algèbre de von Neumann de $R$. On a alors

$$
C(R)=\tau(R)+\beta_{1}(R)+1
$$

pour toute relation ergodique II $_{1}$ de coût fini (voir [4], [5]), et il n'y a pas d'exemples connus pour lesquels $\tau(R)>0$. Le taux de cycles a été introduit dans [4] conjointement avec une technique d'effaçage de cycles, qui permet de faire diminuer la dimension $\operatorname{dim}_{L R} \overline{Z_{1}\left(\Sigma_{\Phi}\right)}$. En fait (cf. [4]) il est possible d'effacer récursivement tous les cycles d'un graphage donné - mais ceci ne suffit pas a priori à entraîner $\tau(R)=0$. Le théorème 1 pourrait fournir une nouvelle approche à ces questions.

Nota Bene. Nous corrigeons ici une erreur typographique qui apparaît deux fois dans la preuve du théorème 4.5 de [3]. Dans la formule centrée $2 C(F) \leq 2+h(R)$, il faut remplacer $h(R)$ par $h_{K}(R)$, et faire de même à la dernière ligne de la preuve. 


\section{Références}

[1] D. Gaboriau, Coût des relations d'équivalence et des groupes. Invent. Math. 139 (2000), 41-98. Zbl 0939.28012 MR 1728876

[2] D. Gaboriau, Invariants $l^{2}$ de relations d'équivalence et de groupes. Publ. Math. Inst. Hautes Études Sci. 95 (2002), 93-150. Zbl 1022.37002 MR 1953191

[3] R. Lyons, M. Pichot, and S. Vassout, Uniform non-amenability, cost, and the first $l^{2}$-Betti number. Groups Geom. Dyn. 2 (2008), 595-617. Zbl 05368765 MR 2442947

[4] M. Pichot, Quasi-périodicité et théorie de la mesure. Ph.D. Thesis, École Normale Supérieure de Lyon, Lyon 2005.

http ://www.umpa.ens-lyon.fr/ gaboriau/Theses/Mikael-Pichot.pdf

[5] M. Pichot, Semi-continuity of the first $l^{2}$-Betti number on the space of finitely generated groups. Comment. Math. Helv. 81 (2006), 643-652. Zbl 1147.20038 MR 2250857

Received February 9, 2009

M. Pichot, IPMU, University of Tokyo, 3-8-1 Komaba, Tokyo, 153-8914, Japan

E-mail: pichot@ms.u-tokyo.ac.jp

S. Vassout, Institut de Mathématiques de Jussieu and Université Paris 7,

175, rue du Chevaleret, 75013 Paris Cedex, France

E-mail: vassout@math.jussieu.fr 